DOI 10.1007/s00417-005-1123-z

\author{
Marc Zaninetti \\ Alexandros N. Stangos \\ German Abdo \\ Constantin J. Pournaras
}

\section{Cavernous sinus thrombosis elicited by a central retinal vein venous stasis retinopathy}

Received: 30 September 2004

Revised: 19 December 2004

Accepted: 20 December 2004

Published online: 9 March 2005

(C) Springer-Verlag 2005

No financial interest. No grant.

M. Zaninetti - A. N. Stangos

Hôpital Ophtalmique Jules-Gonin,

1004 Lausanne, Switzerland

G. Abdo

Département de radiodiagnostic,

Hôpitaux Universitaires Genevois,

Geneva, Switzerland

C. J. Pournaras $(\bowtie)$

Clinique d'ophtalmologie,

Hôpitaux Universitaires Genevois,

22, rue Alcide-Jentzer,

1211 Geneva 14, Switzerland

e-mail: constantin.pournaras@hcuge.ch

Tel.: +41-22-3828394

Fax: +41-22-3828421

\begin{abstract}
Background: Central retinal vein occlusion is a relatively common retinal disorder in the elderly, and those with cardiovascular or thrombophilic risk factors are at increased risk. Although still unsatisfying, some treatments for the acute and chronic phases have been established based on randomized studies. However, for rare conditions mimicking central retinal vein occlusion, treatment of the acute phase should be targeted at etiology. A rare condition mimicking central retinal vein occlusion in a 70-year-old man is presented and discussed. Methods: A 70-yearold man was admitted to the hospital for isovolemic hemodilution related to a central retinal vein occlusion of the elderly, after a sudden decrease in visual acuity to 0.1 . Results: Clinical and laboratory work-up demonstrated a venous stasis retinopathy, related to
\end{abstract}

an atypical cavernous sinus thrombosis of undetermined origin. The patient had experienced in the past 6 months intermittent diplopia and an irreducible conjunctival hyperemia. Hemodilution was dismissed. Soon after initiation of anticoagulation therapy, the patient's clinical signs and symptoms improved. Final visual acuity was 0.8 . Conclusion: Venous stasis retinopathy secondary to cavernous sinus thrombosis is rare. However, careful clinical examination and extensive laboratory work-up is needed to exclude central retinal vein occlusion not associated with common vascular pathologies of the elderly.

Keywords Central retinal vein occlusion - Carotid-cavernous fistula . Hemodilution - Arteriography · Anticoagulation · Proptosis

\section{Introduction}

In the elderly, the principal risk factors for central retinal vein occlusion (CRVO) are arterial hypertension, diabetes mellitus and intraocular hypertonia [9]. However, central retinal vein venous stasis retinopathy mimicking CRVO occasionally results from disease affecting the venous drainage such as retinal arteriovenous communications, carotid-cavernous fistula, cavernous sinus thrombosis or intraorbital processes. All the above leads to an increase of intraluminal pressure within the central retinal vein and consecutive venous stasis at the peripheral retinal venous circulation. Although lowering blood viscosity and administration of antiaggregating agents is the standard treatment for acute stages of CRVO [4, 5], it should not be carried out until other causes of central retinal vein venous stasis retinopathy can be reasonably discounted, because of the possible risk of aggravating the condition, especially in the case of a carotid-cavernous fistula.

\section{Case report}

We report the case of a 70-year-old man with neither cardiovascular nor thrombophilic risk factors who was referred to the clinic by his ophthalmologist for isovolemic hemodilution for CRVO. He mainly complained of a recently sudden loss of visual acuity of the right eye (OD); 


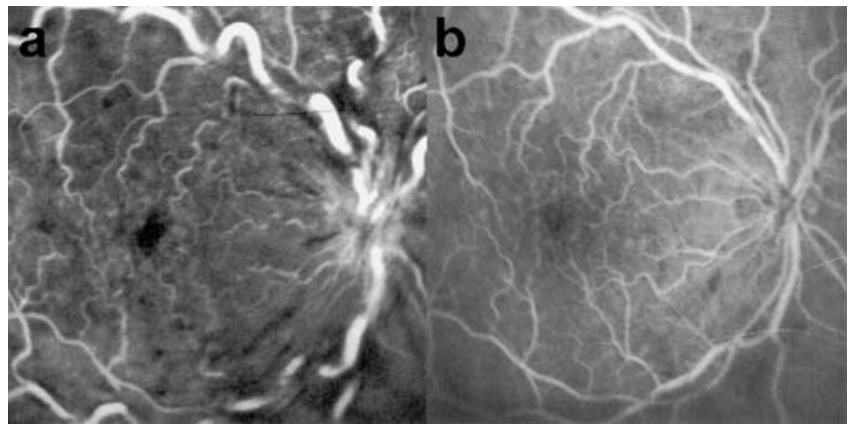

Fig. 1 a Fluorescein angiography of the right eye showing predominating tortuous and dilated retinal veins in the acute phase. b Fluorescein angiography of the right eye showing regression of tortuous and dilated retinal veins and hemorrhages in the remitting phase

he had, however, experienced in the past 6 months two episodes of painless diplopia, each one lasting less than 2 weeks. These episodes were attributed to sinusitis by a general ophthalmologist. The patient was also concerned by a recurrent conjunctival hyperemia resistant to anti-inflammatory drops. No orbital murmur could be retrieved from anamnestic data and clinical examination. The examination revealed visual acuity (VA) OD of 0.1 , a striking dilation of the conjunctival/episcleral vessels, proptosis (Hertel: 13-109-9) and a slowly reactive pupil. The intraocular pressure was slightly asymmetrical $(17 \mathrm{mmHg}$ OD;
$14 \mathrm{mmHg}$ OS). The OD fundus presented with tortuous and dilated vessels, intraretinal hemorrhages (Fig. 1a) and a macular edema. The arteriovenous time was severely delayed at fluorescein angiography. The superior ophthalmic vein was of normal caliber on ultrasound. Orthoptic tests indicated a mechanical limitation of extraocular muscles, but without any nerve palsies. Laboratory testing was negative for any thrombophilic disorders. At this time, it was decided to dismiss the isovolemic hemodilution because the clinical appearance strongly suggested a carotid-cavernous fistula or an intraorbital process, and orbital and cerebral imaging was ordered. CT imaging showed a right orbital blood stasis without mass effect, whereas angio-MRI showed a delayed right cavernous sinus perfusion. Moreover, cerebral arteriography showed lack of opacification of the right sinus cavernosus, yielding a right cavernous sinus thrombosis as the probable diagnosis (Fig. 2a1-2, b1-2). Since no imaging techniques could elicit a carotid-cavernous fistula, we considered it as excluded. A low-molecularweight heparin anticoagulation promptly followed by a coumarin anticoagulation with INR between 2.5 and 3.5 was immediately started by the neurologist. In a few weeks, the conjunctival/episcleral vessel dilation regressed and the fundus clinical picture returned to almost normal (Fig. 1b), except for the persistence of a moderate macular edema, which vanished after a single intravitreal administration of triamcinolone. VA OD, however, remained low because of an advancing cataract, but increased to 0.8 after cataract
Fig. 2 a Arteriography showing injection of contrast medium into the left common carotid artery (1 lateral, 2 anteroposterior). Note the opacification of the left cavernous sinus (arrows). b Arteriography showing injection of contrast medium into the right common carotid artery (1 lateral, 2 anteroposterior). Note the lack of opacification of the right cavernous sinus (arrows)
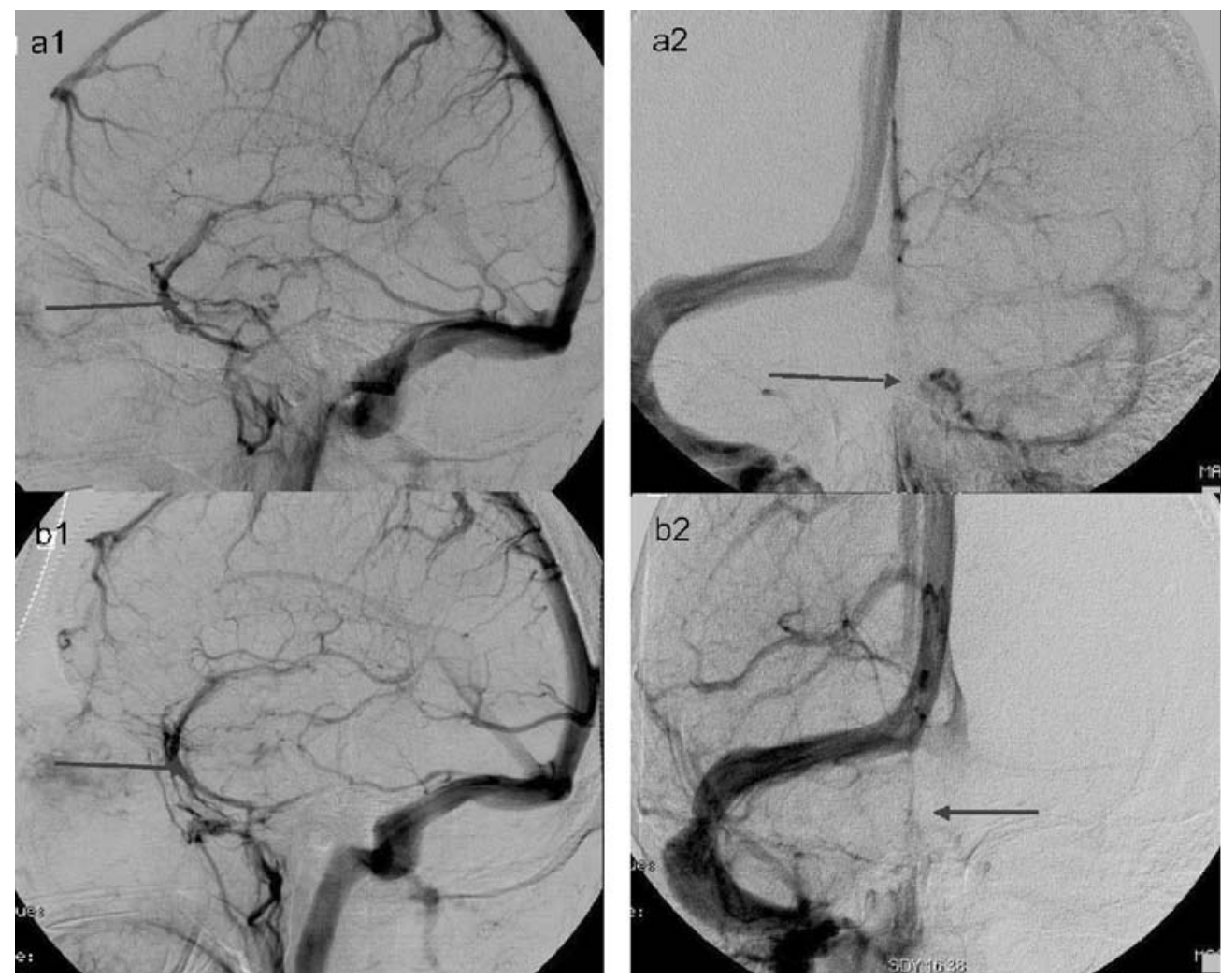
extraction 2 months later. At the same time, angio-MRI showed no delay anymore of the right sinus cavernous perfusion. After a 4-month course, the anticoagulation was stopped and replaced by low-dose aspirin.

\section{Discussion}

Several reports discuss the occurrence of cavernous sinus thrombosis associated with CRVO $[1,2,8]$. However, the case presented here differs in several aspects from the previous reports and from classical cavernous sinus thrombosis. A classical cavernous sinus thrombosis has an acute onset which may be accompanied by an alteration of consciousness. Even if the symptoms are milder, a precipitating event can generally be found, either a contiguous infection or a head trauma or head surgery within a few days to weeks before. Our patient, however, developed varying symptoms at varying intervals, no one symptom being at first suggestive of a cavernous sinus thrombosis, and denied any previous head trauma. Despite this very atypical history, the diagnosis of cavernous sinus thrombosis remains likely for the following reasons: CT and MRI showed an orbital blood engorgement without any other tissue abnormalities, thus the process was purely intravascular. Extensive serological and blood testing did not yield results positive for sarcoidosis and other orbital or cavernous sinus diseases. Cerebral arteriography, corroborating MRI angiographic sequences, showed a lack of perfusion of the right cavernous sinus. Lastly, the patient began to recover quickly after the introduction of an anticoagulation therapy.

We thus conjecture that a previous sinus infection caused some months earlier a low-grade septic cavernous sinus thrombosis, which evolved into a predominant orbital ve- nous hypertension, generating signs and symptoms related to ischemia or tissue engorgement. Finally, after a time lapse of orbital venous hypertension, the blood-retina barrier broke down, causing a central retinal vein venous stasis retinopathy. A number of cases of central retinal vein venous stasis retinopathy have been described after carotidcavernous fistula (e.g., [3, 7]), far more often than after cavernous sinus thrombosis. However, venous sinus hypertension and venous sinus thrombosis may lead to indirect arteriovenous fistula [6]. One can thus imagine that cavernous sinus thrombosis of the type presented here could have occurred more often, but would have evolved before diagnosis into an indirect carotid-cavernous fistula, or alternatively the thrombosis would have spontaneously resolved.

Isovolemic hemodilution is the proposed treatment for the acute phase of CRVO. However, in conditions mimicking CRVO but not being classical CRVO, this treatment has not been studied. Even if it could be relatively safe in central retinal vein venous stasis retinopathy caused by a cavernous sinus thrombosis, it would, however, possibly be thought unsafe in the case of a carotid-cavernous fistula, since the hemodynamic state would allow increasing flow into the fistula. In the case presented here, postponing hemodilution was of prime importance since the suspicion of carotid-cavernous fistula was primarily high.

Although rare in the elderly, central retinal vein venous stasis retinopathy of this type should not be confused with a classical CRVO, since the treatment of the acute phase might be targeted according to the etiology. It is thus of importance to carry out a careful clinical examination and, if needed, extensive laboratory work-up for untypical history of CRVO.

\section{References}

1. Boniuk M (1972) The ocular manifestations of ophthalmic vein and aseptic cavernous sinus thrombosis. Trans Am Acad Ophthalmol Otolaryngol 76:1519-1534

2. Brismar G, Brismar J (1977) Aseptic thrombosis of orbital veins and cavernous sinus. Acta Ophthalmol 55:9-22

3. Brunette I, Boghen D (1987) Central retinal vein occlusion complicating spontaneous carotid-cavernous fistula. Arch Ophthalmol 105:464-465
4. Glacet-Bernard A, Zourdani A, Milhoub M, Maraqua N, Coscas G, Soubrane G (2001) Effect of isovolemic hemodilution in central retinal vein occlusion. Graefes Arch Clin Exp Ophthalmol 239:909-914

5. Hansen LL, Wiek J, Wiederholt M (1989) A randomised prospective study of treatment of non-ischaemic central retinal vein occlusion by isovolaemic haemodilution. Br J Ophthalmol 73:875-879

6. Herman JM, Spetzler RF, Bederson JB, Kurbat JM, Zabramski JM (1995) Genesis of a dural arteriovenous malformation in a rat model. J Neurosurg $83: 539-545$
7. Komiyama M, Yamanaka K, Nagata Y, Ishikawa H (1990) Dural carotid-cavernous sinus fistula and central retinal vein occlusion: a case report and a review of the literature. Surg Neurol 34:255-259

8. Sharma S, Cruess AF (1996) Central retinal vein occlusion associated with cavernous sinus thrombosis. Can J Ophthalmol 31:255-256

9. The Eye Disease Case-Control Study Group (1996) Risk factors for central retinal vein occlusion. Arch Ophthalmol 114:545-554 